\title{
Meditação na universidade: a motivação de alunos da UFRGS para aprender meditação
}

\author{
Carolina Baptista Menezes \\ Bruna Fiorentin \\ Lisiane Bizarro
}

\begin{abstract}
Resumo
O treinamento em meditação sentada e silenciosa tem sido cada vez mais usado em contextos educacionais. Este estudo objetivou conhecer o interesse e a motivação de universitários para participar de um treino de meditação sentada e silenciosa. Foi realizado um levantamento online que utilizou um questionário sociodemográfico com uma pergunta aberta sobre a motivação para participar do treino e o Self-Report Questionnaire para identificar transtornos psiquiátricos menores. Participaram 524 universitários de 42 cursos ( $M=25$ anos, $D P=5,16,64,3 \%$ mulheres). A maioria era solteiro (88\%), com renda entre 1 e 5 salários mínimos (51,8\%), de diferentes religiões $(n=20)$ e sem sintomas psiquiátricos (73\%). Os principais motivos para treinar meditação foram os benefícios emocionais (25,59\%), gosto e interesse pelo assunto $(24,67 \%)$, benefícios cognitivos $(16,02 \%)$ e curiosidade e interesse na pesquisa $(15,11 \%)$. O interesse pela meditação não está restrito a universitários com características sociodemográficas específicas, com determinada crença ou problemas psiquiátricos.
\end{abstract}

Palavras-chave: Meditação, intervenção psicológica, estudantes universitários.

\section{Meditation on university campus: motivation of UFRGS students to learn}

\author{
how to meditate
}

\begin{abstract}
Sitting and silent meditation training has been increasingly used in educational contexts. In this study we propose to investigate the interest and motivation of undergraduate students to take part in a sitting and silent meditation training. An online survey has been carried out, using a sociodemographic questionnaire with an open question about the motivation to participate in the training, and the Self Report Questionnaire to identify minor psychiatric disorders. 524 students, from 42 courses participated ( $M=25$ years, $D P=5,16,64,3 \%$ female). Most were single (88\%), with an income between 1 and 5 minimum wage (51,8\%), from different religions $(n=20)$, and without psychiatric symptoms (73\%). The main reasons for training meditation were emotional benefits $(25,59 \%)$, interest in the subject $(24,67 \%)$, cognitive benefits $(16,02 \%)$, and curiosity and interest in the research proposal $(15,11 \%)$. The interest in meditation is not restricted to undergraduates with specific sociodemographic characteristics or with a certain belief or psychiatric problems.
\end{abstract}

Keywords: Meditation, psychological intervention, college students.

\section{Meditación en la universidad: motivación de alumnos de la universidad Federal de Rio Grande del Sur (UFRGS) para aprender meditación}

\section{Resumen}

El entrenamiento de meditación sentada y silenciosa ha sido cada vez más utilizado en contextos educacionales. Este estudio tuvo el objetivo de conocer el interés y la motivación de universitarios para participar de una práctica de meditación sentada y silenciosa. Se realizó una encuesta online compuesta por un cuestionario socio-demográfico con una pregunta abierta sobre la motivación para participar de la práctica. Además se utilizó el Self-Report Questionnaire para identificar trastornos psiquiátricos menores. Participaron 524 universitarios de 42 cursos $(P=25$ años, $D S=5,16,64,3 \%$ mujeres). La mayoría era soltero (88\%), con renta entre 1 y 5 sueldos mínimos $(51,8 \%)$, de diferentes religiones $(n=20)$ y sin síntomas psiquiátricos (73\%). Los principales motivos para practicar meditación fueron los beneficios emocionales (25,59\%), gusto e interés por el tema $(24,67 \%)$, beneficios cognitivos $(16,02 \%)$ y curiosidad e interés en la investigación $(15,11 \%)$. El interés por la meditación no se restringe a universitarios con características socio-demográficas específicas, con determinada creencia o problemas psiquiátricos.

Palabras clave: Meditación, intervención psicológica, estudiantes universitarios. 


\section{Introdução}

Alguns estudos apontam que a fase universitária pode ser marcada por algumas dificuldades, dentre elas acadêmicas, interpessoais e pessoais. Por exemplo, dados mostram que há um alto consumo de álcool e drogas entre essa população (Domingos \& Domingos, 2005) e que a sua qualidade de vida, especialmente nos domínios físico, psicológico, nível de independência e ambiente, é pior em comparação à amostra utilizada para a validação do instrumento utilizado (WHOQOL) (Cerchiari, 2004).

Além disso, ao avaliar a prevalência de transtornos mentais comuns em estudantes de medicina através do Self-Report Questionnaire - SRQ, um estudo encontrou que quase metade dos alunos obteve pontuação que os classifica como possíveis casos de transtornos mentais comuns. Esta pontuação se associou com as variáveis "não recebe apoio emocional que precisa", "tem dificuldade para fazer amigos" e "pensa em abandonar o curso" (Lima, Domingues, \& Cerqueira, 2006). Embora com prevalência menor (ao redor de $25 \%$ ), alunos de outros cursos também obtêm pontuações que indicam a presença de transtornos mentais menores, ou sintomas relacionados (Cerchiari, 2004; Gastaud e cols., 2006).

Por outro lado, muitas vezes os próprios alunos reconhecem que necessitam de maior assistência por parte da universidade (Bolsoni-Silva, Loureiro, Rosa, \& Oliveira, 2010; Oliveira, Dantas, Azevedo, \& Banzato, 2008). Em um período de 17 anos de procura pelo serviço de saúde mental oferecido pela instituição UNICAMP, foi observado que $75 \%$ da demanda foi feita por alunos de graduação, em comparação a $24,4 \%$ de alunos de pós-graduação. As queixas mais frequentes foram dificuldades de relacionamentos interpessoais, conflitos parentais e desempenho acadêmico pobre (Oliveira e cols., 2008). Alguns autores propõem que mais intervenções assistenciais deveriam ser promovidas pelas instituições de ensino, tal como o treino de habilidades sociais (Bolsoni-Silva e cols., 2010), ou uma maior preparação para a transição da fase universitária para o mercado de trabalho (Teixeira \& Gomes, 2004).

Nas duas últimas décadas tem havido um aumento no uso de intervenções em contextos educacionais que utilizam a meditação sentada e silenciosa como ferramenta principal, especialmente nos Estados Unidos. A disseminação dessa prática deve-se ao fato de que ela envolve um tipo de treinamento mental que busca desenvolver e aprimorar habilidades mentais - cognitivas e emocionais - que podem ser benéficas para um desenvolvimento saudável e desempenho mais eficiente, tanto no que tange a aspectos intra como interpessoais (para uma revisão da operacionalização do conceito e de sua inserção no campo da psicologia, ver Cardoso, Souza, Camano, \& Leite, 2004; Menezes \& Dell'Aglio, 2009).

Instituições destinadas a promover tais iniciativas, assim como encontros para discutir as mesmas e suas avaliações, principalmente no âmbito científico, já existem. Alguns exemplos são o Mind and Life Institute - MLI (http:// www.mindandlife.org), sendo alguns de seus encontros intitulados "Educação, Neurociência do Desenvolvimento e Práticas Contemplativas: Perguntas, Desafios e Oportunidades", e "Educação dos Cidadãos do Mundo para o Século 21" (http://www.educatingworldcitizens.org/mlern.html). Outras associações são a Mindfulness in Education Network - MiEN (http://www.mindfuled.org) e o Center for Contemplative Mind in Society (http://www.contemplativemind.org). Uma descrição mais detalhada de cada um, assim como relatórios e materiais de apoio podem ser encontrados nos respectivos sites.

Dentre os relatos científicos que abordam intervenções no ensino fundamental, alguns exemplos incluem o programa BREATHE (Broderick \& Stacie, 2009), o qual trabalha com grupos de nona e décima série após o período escolar; programas de onze ou doze semanas de ioga para adolescentes (Khalsa, Hickey-Schultz, Cohen, Steiner, \& Cope, 2011), para alunos de escolas de nível socioeconômico baixo (Mendelson e cols., 2010) e como uma atividade de férias (Manjunath \& Shirley, 2004); a intervenção MAPs (Mindful Awareness Practices), com oito semanas de duração, destinada a crianças (Flook e cols., 2010) e adolescentes com transtorno de déficit de atenção e hiperatividade (TDAH) (Zylowska e cols., 2008); além de um programa para professores, o Inner Resilience Program (Lantieri \& Goleman, 2008), o qual inclui encontros semanais com os educadores, visando auxiliar no manejo do estresse através de práticas de ioga e meditação, encontros para diálogos que proporcionem maior integração entre a equipe, retiros de fim de semana, além de oficinas voltadas ao desenvolvimento profissional.

Em todas essas iniciativas foram demonstrados resultados favoráveis ao uso da prática de meditação no contexto educacional, tais como redução de afeto negativo e aumento nos sentimentos de calma, relaxamento e autoaceitação (Broderick \& Stacie, 2009); controle de raiva e menor fadiga (Khalsa e cols., 2011); redução da ruminação, pensamentos intrusivos e reatividade emocional (Mendelson e cols., 2010); incremento em funções executivas, em especial nas facetas de regulação do comportamento, metacognição e controle executivo global (Flook e cols., 2010); redução no relato de sintomas de TDAH, assim como uma melhora no desempenho das tarefas comportamentais de atenção (Zylowska e cols., 2008); melhora no desempenho em um teste de memória, no subtipo espacial, em que as crianças tinham que reproduzir o maior número possível de figuras geométricas que haviam sido projetadas em diversos slides (Manjunath \& Shirley, 2004); além de redução dos sintomas de estresse, aumento nos escores de mindfulness e aumento da confiança interpessoal entre os professores (Lantieri \& Goleman, 2008).

$\mathrm{Na}$ universidade, iniciativas semelhantes são realizadas. Uma que merece ser mencionada, mesmo que não tenha resultados publicados ainda, é realizada na universidade de Brown (http://med.brown.edu/education/concentrations/contemplative.html). Lá, existe um programa integrado ao currículo, sendo oferecido como uma das ênfases que 
os alunos podem escolher na sua formação. Esse envolve disciplinas teóricas como a neurociência da meditação e as filosofias que a originaram, além de encontros práticos, denominados "laboratório de meditação", onde se experimentam diversas técnicas. Um dos resultados práticos dessa iniciativa foi o surgimento da casa de estudantes Cheetah (http://www.cheetahhouse.org/) nessa universidade, onde moram alunos universitários engajados na prática da meditação e que buscam cada vez mais integrá-la nas suas vidas, em diferentes âmbitos. Um dos objetivos da casa é incentivar e desenvolver nos moradores um papel social ativo, com atuação em projetos comunitários. Essas e outras informações encontram-se nas páginas de internet citadas.

Outras intervenções são geralmente mais curtas e pontuais. Entre elas, um treino de três meses de meditação transcendental oferecido como atividade extracurricular (Tanner e cols., 2009); o programa de redução de estresse baseado em mindfulness (Mindfulness-Based Stress Reduction Program-MBSR) e o programa de oito pontos de Easwaran (Eight Point Program-EPP) (Jain e cols., 2007; Shapiro, Oman, Thoresen, Plante, \& Flinders, 2008).

Da mesma forma que no ensino fundamental, resultados positivos e estatisticamente significativos foram encontrados, incluindo um aumento nas quatro facetas - observação, descrição, atividades com consciência e aceitação sem julgamento - do construto mindfulness medido pelo The Kentucky Inventory of Mindfulness Skill (Tanner e cols., 2009); um aumento significativo de mindfulness medido pela Mindful Attention and Awareness Scale (MAAS) (Shapiro, Oman e cols., 2008); e redução do estresse, aumento nos estados positivos do humor e redução da distração e da ruminação (Jain e cols., 2007).

A inclusão da meditação na formação de alunos, seja através de práticas extracurriculares, ou integradas ao próprio currículo, pode ser benéfica para a formação acadêmica e pessoal do estudante. Além disso, as pesquisas relatam uma boa adesão, com baixa taxa de perdas, e que os alunos, pais, professores e instituições se interessam e apoiam este tipo de atividade. No Brasil, já existem pesquisas sobre meditação, tal como as realizadas na Universidade Federal de São Paulo - UNIFESP (Cardoso e cols., 2004; Leite e cols., 2010), ou na Universidade Federal do Rio Grande do Sul (Menezes \& Dell'Aglio, 2010). No entanto, ainda se observa uma ausência da aplicação da meditação, ou relato da mesma, no contexto educacional. Também não há relatos a respeito do conhecimento sobre ou interesse pela meditação entre estudantes universitários, o que seria um primeiro passo para viabilizar o planejamento de intervenções. O objetivo deste trabalho foi investigar se há interesse dos alunos da Universidade Federal do Rio Grande do Sul (UFRGS) em participar em um treino extracurricular de seis semanas de meditação e, se sim, quais são as suas características sociodemográficas, seu curso e semestre, se há queixas de sintomas psiquiátricos menores e qual a sua motivação para aprender meditação.

\section{Método}

Este foi um estudo inicial, caracterizado como um levantamento de caráter exploratório e descritivo. O mesmo faz parte de uma pesquisa mais ampla, a qual visa oferecer um treino de meditação sentada e silenciosa aos alunos da UFRGS como uma atividade extracurricular. A ideia é que as técnicas ensinadas auxiliem os alunos a exercer e aprimorar aspectos relacionados à atenção e à regulação emocional.

\section{Participantes}

Participaram do levantamento 524 alunos de graduação de diversos cursos da UFRGS. Ao serem convidados a participar, os alunos eram informados que deveriam ter entre 19 e 40 anos, não ter experiência prévia em meditação, não ter diagnóstico atual de transtorno mental, não estar fazendo uso de medicação psicoativa e/ou tratamento psicoterápico. Esses critérios foram estipulados tendo em vista o objetivo do estudo mais amplo. A descrição da amostra encontra-se na Tabela 1 e 2.

\section{Instrumentos}

Questionário Sociodemográfico: Questionário elaborado para a pesquisa a fim de investigar dados sociodemográficos, tais como sexo, idade, renda, estado civil, assim como curso, semestre, uso de medicação psicoativa, tratamento psiquiátrico ou psicoterapêutico e religião. Também foi incluída uma questão aberta, que perguntava "por que motivos você se interessou e gostaria de participar deste treinamento?".

Self-Report Questionnaire - SRQ (Harding e cols., 1980): Questionário de identificação de sintomas psiquiátricos em nível de atenção primária que já foi validado para a população brasileira (Mari \& Willians, 1986). Composto por 20 questões elaboradas para detecção de sintomas neuróticos e 3 para sintomas psicóticos. O ponto de corte utilizado tem sido seis ou mais respostas afirmativas para homens, e sete ou mais respostas afirmativas para mulheres. Para o presente levantamento, os respondentes que ultrapassaram o ponto de corte foram incluídos.

\section{Procedimento}

A divulgação da pesquisa foi realizada através de um e-mail enviado para as Comissões de Graduação (Comgrads) de todos os cursos da UFRGS, as quais eram solicitadas a encaminhá-lo à lista de alunos de seu curso, e de 20 cartazes colados por diversos prédios. O e-mail convidava os alunos a participarem de um treino gratuito de meditação, a ser realizado na própria universidade, como atividade extracurricular, parte de uma pesquisa de doutorado. Era informado que, para participar, os interessados deveriam, 
inicialmente, responder a algumas perguntas, as quais poderiam ser acessadas online, através de um link fornecido no próprio corpo do e-mail. Ao acessá-lo, a primeira página apresentava o Termo de Consentimento Livre e Esclarecido, sendo a marcação do item de aceitação para participar indispensável para que os respondentes conseguissem acessar as perguntas subsequentes. Na segunda página constava o questionário sociodemográfico e, na terceira, o SRQ. Ao final, uma página de agradecimento era exibida. O projeto foi aprovado pelo Comitê de Ética em Pesquisa do Instituto de Psicologia da UFRGS. Através de alunos que procuraram a pesquisa pelos cartazes, soube-se que alguns cursos não receberam o e-mail com a divulgação da pesquisa. Não foi feito um acompanhamento com as Comgrads para averiguar quais não encaminharam o e-mail.

\section{Análise dos Dados}

Para a descrição da amostra foram utilizadas estatísticas descritivas, através do cálculo de média, desvio padrão e porcentagem. Para a análise dos motivos, os dados foram analisados por meio da análise de conteúdo quantitativa (Bardin, 1977), de forma exploratória, já que não foram criadas categorias a priori. Esta foi realizada a partir de três etapas principais: a pré-análise, a exploração do material e o tratamento dos resultados e interpretação. Na pré-análise, os conteúdos foram lidos e organizados. Na exploração do material, realizou-se a codificação, a qual passou por três fases: a) a transformação dos conteúdos em unidades de análise; b) a definição das categorias a partir das semelhanças e diferenças entre as unidades; e c) a enumeração das

Tabela 1. Distribuição de variáveis sociodemográficas, religião e sintomas psiquiátricos menores.

\begin{tabular}{|c|c|c|}
\hline & $\mathrm{N}$ & $\mathrm{M}(D P)$ \\
\hline Idade* & 483 & $\begin{array}{l}25,06 \\
(5,16)\end{array}$ \\
\hline & & $\%$ \\
\hline $\begin{array}{l}\text { Sexo* } \\
\text { Feminino } \\
\text { Masculino }\end{array}$ & $\begin{array}{l}314 \\
174\end{array}$ & $\begin{array}{l}64,3 \% \\
35,7 \%\end{array}$ \\
\hline $\begin{array}{l}\text { Estado Civil* } \\
\text { Solteiro/a } \\
\text { Casado/a } \\
\text { Divorciado/a }\end{array}$ & $\begin{array}{c}426 \\
51 \\
4\end{array}$ & $\begin{array}{c}88 \% \\
10,5 \% \\
0,8 \%\end{array}$ \\
\hline $\begin{array}{l}\text { Renda* }^{*} \\
<1 \text { salário mínimo } \\
\text { Entre } 1 \text { e } 5 \text { salários } \\
\text { Entre } 5 \text { e } 10 \text { salários } \\
\text { Entre } 10 \text { e } 15 \text { salários } \\
>\text { de } 15 \text { salários }\end{array}$ & $\begin{array}{c}163 \\
249 \\
49 \\
11 \\
2\end{array}$ & $\begin{array}{c}33,9 \% \\
51,8 \% \\
10,2 \% \\
2,3 \% \\
0,4 \%\end{array}$ \\
\hline $\begin{array}{l}\text { Religião* } \\
\text { Cristãos Católicos (Católica, Cristã) } \\
\text { Espírita } \\
\text { Cristãos Protestantes (Evangélicos, Testemunha de Jeová, Luteranos, Metodistas, } \\
\text { Protestantes, Mórmons) } \\
\text { Crença Espiritual Não Religiosa } \\
\text { Afro-brasileiras (Afro-brasileira, Candomblé, Umbanda) } \\
\text { Agnóstico } \\
\text { Orientais (Hindu, Budista) } \\
\text { Judaica } \\
\text { Deísta } \\
\text { Pagões (Pagã, Wicca) }\end{array}$ & $\begin{array}{c}119 \\
52 \\
20 \\
\\
9 \\
6 \\
6 \\
4 \\
4 \\
4 \\
3\end{array}$ & $\begin{array}{c}52,2 \% \\
23,1 \% \\
8,8 \% \\
\\
4,0 \% \\
2,6 \% \\
2,6 \% \\
1,8 \% \\
1,8 \% \\
1,8 \% \\
1,3 \%\end{array}$ \\
\hline $\begin{array}{l}\text { Sintomas no SRQ* } \\
0 \\
1-2 \\
3-5 \\
6-7 \\
8-11 \\
12-14 \\
15-18\end{array}$ & $\begin{array}{c}113 \\
83 \\
120 \\
74 \\
91 \\
43 \\
13\end{array}$ & $\begin{array}{c}21,4 \% \\
15,9 \% \\
22,7 \% \\
13,1 \% \\
16,2 \% \\
8,3 \% \\
2,5 \%\end{array}$ \\
\hline
\end{tabular}

Nota. * o máximo de valores ignorados foi 297. 
unidades dentro de cada categoria, pela sua frequência de aparição. Com base na distribuição das unidades, também se calculou a frequência de cada categoria.

\section{Resultados}

A descrição da amostra encontra-se nas Tabelas 1 e 2. O interesse pelo treino foi por alunos de todos os semestres, diferentes cursos $(n=42)$, agrupados em cinco grandes áreas, bem como religiões $(n=20)$, igualmente agrupadas em tipos principais. Também abrangeu pessoas sem queixas de sintomas psiquiátricos menores (73\%), ou seja, homens e mulheres com menos de seis e sete pontos, respectivamente, e com queixas de sintomas psiquiátricos (27\%), isto é, homens e mulheres com escore a partir de seis e sete pontos, respectivamente.

Tabela 2. Distribuição do semestre e curso

\begin{tabular}{|l|c|c|}
\hline & $\mathrm{N}$ & $\%$ \\
\hline Semestre* $^{*}$ & & \\
2 & 61 & $14,8 \%$ \\
3 & 38 & $9,2 \%$ \\
4 & 68 & $16,5 \%$ \\
5 & 52 & $12,6 \%$ \\
6 & 69 & $16,7 \%$ \\
7 & 27 & $6,6 \%$ \\
8 & 47 & $11,4 \%$ \\
9 & 23 & $5,6 \%$ \\
10 & 18 & $4,5 \%$ \\
11 & 8 & $1,9 \%$ \\
\hline & 1 & $0,2 \%$ \\
\hline Curso* & & \\
Ciências Humanas e Artes & 180 & $40,3 \%$ \\
Ciências Exatas & 74 & $16,5 \%$ \\
Educação, Letras & 71 & $16,0 \%$ \\
Ciências Naturais & 70 & $15,6 \%$ \\
Ciências da Saúde & 50 & $11,6 \%$ \\
\hline
\end{tabular}

Nota. * o máximo de valores ignorados foi 112

Quanto à questão aberta sobre os motivos, foram levantadas 543 respostas, as quais foram categorizadas em 10 domínios: benefícios emocionais, gosto e interesse pelo assunto, benefícios cognitivos, curiosidade e interesse na proposta, motivação para ajudar na pesquisa, aprender e experienciar algo novo, indicação de alguém, ainda não tinha tido oportunidade, aspecto não religioso e outro.

$\mathrm{Na}$ categoria benefícios emocionais, os participantes salientaram a vontade de aprender a lidar melhor com aspectos como ansiedade, estresse e depressão e adquirir maior qualidade de vida. Na categoria gosto e interesse pelo assunto, as pessoas referiram gostar do assunto da meditação e ter sempre tido interesse pelo mesmo, assim como vontade de aprender mais a respeito. Na categoria benefícios cognitivos, os respondentes relataram uma motivação voltada para o aperfeiçoamento de habilidades como concentração e atenção. No domínio curiosidade e interesse na proposta, foi ressaltado que a ideia da pesquisa despertou curiosidade e interesse. No grupo de respostas motivação para ajudar na pesquisa, os participantes relataram a sua vontade de colaborar com pesquisas científicas, salientando a importância das mesmas. Na categoria aprender e experiencar algo novo ficaram as respostas com ênfase na vontade de ter uma nova experiência e adquirir um novo conhecimento. No domínio indicação de alguém, foram incluídas respostas sobre a influência de outra pessoa na sua busca e interesse pela meditação. Alguns também relataram que nunca tinham tido uma oportunidade para meditar, e suas respostas foram agrupadas em ainda não tinha tido oportunidade. No grupo do aspecto não religioso, alguns respondentes disseram ficar atraídos por um treino de meditação que não tivesse envolvimento com alguma filosofia ou religião particular. Outro diz respeito a respostas que não se relacionaram a nenhuma das categorias criadas. É importante ressaltar que as análises contemplaram o total de respostas ( $n=543$ ) e não o total de pessoas, visto que cada participante podia apresentar mais de uma resposta. As categorias e suas frequências, bem como as respostas mais citadas em cada categoria, estão apresentadas na Tabela 3.

\section{Discussão}

O objetivo deste levantamento foi explorar se os alunos da UFRGS teriam interesse em fazer um treino de meditação extracurricular, assim como as características desses interessados e qual a sua motivação para participar. Muitos alunos $(n=524)$ responderam ao levantamento mostrando-se interessados em fazer parte da pesquisa. Observa-se que não existe um perfil específico. O interesse em aprender meditação foi tanto de homens, quanto mulheres, assim como de adultos jovens e médios. Analogamente à idade, pessoas de todos os semestres, embora com uma concentração maior na primeira metade do curso, mostraram-se interessadas, indicando que programas como esse podem ser oferecidos em diferentes etapas da formação universitária. Sugere-se que pode ser útil investigar se há algum período em que o aproveitamento seja maior, tal como no início da formação, quando o aluno ainda está se adaptando ao curso e às novas exigências, ou ao final, quando é preciso lidar com a busca de emprego e a entrada no mercado de trabaIho (Teixeira \& Gomes, 2004).

Da mesma forma que o semestre, apesar de haver maior frequência de determinados cursos, de forma geral observa-se uma diversidade. Portanto, parece haver interesse de que tais programas sejam oferecidos para alunos de qualquer área, podendo ser caracterizados como uma 
Tabela 3. Percentuais de cada categoria e de suas respostas mais frequentes.

\begin{tabular}{|c|c|c|}
\hline Categorias e respostas & $\mathrm{N}$ & $\%$ \\
\hline Benefícios emocionais & 139 & $25,59 \%$ \\
\hline Reduzir estresse/ansiedade, acalmar, relaxar & 51 & $36,69 \%$ \\
\hline Qualidade de vida/bem-estar mental & 36 & $25,89 \%$ \\
\hline Interesse pela psicologia/mente/cérebro & 26 & $18,71 \%$ \\
\hline Autoconhecimento/autocontrole & 26 & $18,71 \%$ \\
\hline Gosto e interesse pelo assunto & 134 & $24,67 \%$ \\
\hline Sempre teve interesse por meditação & 74 & $55,22 \%$ \\
\hline Quer conhecer/aprender mais sobre meditação & 24 & $17,92 \%$ \\
\hline Gosta do assunto, chama atenção, parece bacana & 17 & $12,68 \%$ \\
\hline Interesse porque já lê/ouve falar nos benefícios & 16 & $11,95 \%$ \\
\hline Outros & 3 & $2,23 \%$ \\
\hline Benefícios cognitivos & 87 & $16,02 \%$ \\
\hline Dificuldades e vontade de melhorar atenção/foco/concentração & 69 & $79,31 \%$ \\
\hline Melhorar rendimento no estudo/trabalho & 18 & $20,69 \%$ \\
\hline Curiosidade e interesse na proposta da pesquisa & 82 & $15,11 \%$ \\
\hline Curiosidade & 55 & $67,07 \%$ \\
\hline Achou a pesquisa/ideia interessante & 27 & $32,93 \%$ \\
\hline Motivação para ajudar na pesquisa & 57 & $10,49 \%$ \\
\hline Vontade de colaborar com a pesquisa & 51 & $89,47 \%$ \\
\hline Acredita que pesquisas são válidas/importantes & 6 & $10,53 \%$ \\
\hline Aprender e experienciar algo novo & 18 & $3,31 \%$ \\
\hline Por ser uma experiência nova/querer aprender coisas novas & 18 & $100 \%$ \\
\hline Indicação de alguém & 12 & $2,21 \%$ \\
\hline Conversou com alguém que indicou & 7 & $58,34 \%$ \\
\hline Teve algum contato em alguma disciplina & 5 & $41,66 \%$ \\
\hline Ainda não tinha tido oportunidade & 7 & $1,29 \%$ \\
\hline Nunca tive oportunidade/chance & 7 & $100 \%$ \\
\hline Aspecto não religioso & 3 & $0,58 \%$ \\
\hline Explicação não religiosa para efeitos da meditação & 3 & $100 \%$ \\
\hline Outro & 4 & $0,73 \%$ \\
\hline Total & 543 & $100 \%$ \\
\hline
\end{tabular}

atividade extracurricular geral, a qual não precisa estar vinculada a uma única graduação ou departamento. Essa configuração inclusive contribui para que alunos da universidade de diferentes formações e que, em geral, não fazem atividades comuns, possam ter um espaço para interação e troca. Dessa forma, seria possível trabalhar a queixa dos graduandos sobre dificuldades de relacionamento (Oliveira e cols., 2008).

Os alunos também relataram uma variedade de religiões, mesmo que algumas menos frequentes, o que indica que a meditação é uma técnica aceita por pessoas de diferentes crenças. Isso provavelmente ocorre porque essa prática é principalmente voltada ao treino de habilidades mentais e ao desenvolvimento psicológico, os quais independem de credo. Por esse motivo, cada vez mais a meditação tem sido incorporada na atuação e investigação em diversas áreas, entre elas ciências sociais, humanas e biológicas (Broderick \& Stacie, 2009; Flook e cols., 2010; Shapiro, Brown e cols., 2008).

Outro achado importante é que o interesse pelo treino foi tanto por alunos sem (73\%) e com queixa de sintomas psiquiátricos menores (27\%), sendo a porcentagem de sintomas nessa amostra semelhante a outros estudos (Cerchiari, 2004; Gastaud e cols., 2006). A busca por quem não tem queixas possivelmente ocorre porque qualquer pessoa pode almejar o desenvolvimento de habilidades cognitivas e emocionais, desejando tornar-se um ser humano sempre mais saudável. A meditação é uma prática com a qual se 
treina autoconsciência, autoconhecimento e controle do processamento mental, especialmente negativo, tal como ruminações (Lutz, Dunne, \& Davidson, 2007), não sendo imprescindível a presença de sintomas para a busca da mesma e de uma maior qualidade de vida. Da mesma forma, para quem enfrenta problemas e sintomas que geram sofrimento, a meditação pode ser igualmente importante, pois as qualidades citadas podem auxiliar no enfrentamento da(s) dificuldade(s), ajudando a amenizá-la(s) ou até a superá-la(s). Nesse sentido, muitos estudos mostram que a prática está associada à redução de sintomas como estresse, ansiedade e depressão (Jain e cols., 2007; Shapiro, Oman e cols., 2008) e, por essa razão, tem sido um componente fundamental de programas voltados ao tratamento de transtornos psiquiátricos específicos, atuando como intervenção complementar (Baer, 2006; Zylowska e cols., 2008).

Com base na distribuição da ocorrência de sintomas entre alunos, assim como o seu interesse pela meditação, entende-se que intervenções baseadas na meditação são uma alternativa para a assistência aos estudantes no contexto universitário. Essas intervenções poderiam ser destinadas a grupos clínicos e não clínicos, e estudos avaliando a sua implementação são necessários.

Além da presença de sintomas psiquiátricos menores, ao avaliar as motivações para participar dessa atividade, observou-se que a busca por benefícios emocionais é a principal. Muitos alunos queixaram-se de ansiedade e estresse, manifestando o interesse em aprender técnicas e estratégias que os auxiliem a lidar melhor com essas emoções e que Ihes proporcionem a vivência de outras mais positivas, como calma, tranquilidade, bem-estar mental/emocional. Sabe-se que o sofrimento psíquico, tal como depressão, ansiedade e baixa autoestima, está entre os fatores que podem desencadear o uso de drogas, o qual é considerado alto entre estudantes universitários (Domingos \& Domingos, 2005). Assim, a redução dessas emoções deveria ser contemplada nos programas de assistência ao aluno. Ao que tudo indica, os estudantes sabem identificar a presença de emoções negativas - uma vez que relataram o desejo de diminuir o estresse e a ansiedade - e demonstram querer experienciar estados mais positivos, mas talvez faltem ferramentas para conseguir fazê-lo.

É igualmente interessante o fato de os benefícios emocionais estarem entre os mais motivadores, visto que, para quem faz meditação, esses também se caracterizam como os mais citados (Menezes \& Dell'Aglio, 2009). Tais dados apoiam a estreita aproximação que tem sido feita entre a prática meditativa e a psicologia (Ekman, Davidson, Ricard, \& Wallace, 2005; Wallace \& Shapiro, 2006; Walsh \& Shapiro, 2006) e sugerem que as pessoas, mesmo sem praticar, associam a meditação ao bem estar.

Possivelmente, por acreditar nessa associação e desejá-la, é que a meditação desperta o interesse de muitos. A segunda categoria de motivação mais frequente envolveu respostas de que as pessoas sempre tiveram algum interesse e gosto pelo assunto, ou que o tema chama a atenção. Assim, o relato de que há interesse pela prática meditativa, juntamente às respostas das categorias de que os participantes nunca tinham tido oportunidade para praticar e de que valorizaram a meditação ser oferecida fora de um contexto filosófico-religioso, apoiam que sejam oferecidos treinos de meditação no contexto universitário. Os alunos têm interesse, estão abertos à proposta e precisam de espaços para se aproximar e experimentar a técnica. Ainda nessa categoria de interesse pela meditação, alguns estudantes mencionaram que esse advém de leituras, o que reforça a importância dos relatos científicos sobre as iniciativas e seus resultados, conforme já apontado na introdução. Tais relatos podem ser proveitosos não só para quem cria as iniciativas, mas também para quem tem vontade de conhecer e usufrui-las.

A terceira categoria mais frequente também contribui para a ideia de inserir treinos de meditação na universidade. Muitos alunos queixaram-se de falta de atenção e concentração, mostrando desejo de melhorar habilidades cognitivas, bem como o seu desempenho no estudo e trabalho, corroborando os dados de Oliveira e cols. (2008). O treino da atenção é um dos aspectos centrais da prática da meditação sentada e silenciosa, e muitos estudos têm mostrado uma melhora dessa função a partir desse tipo de treino (para revisão ver Lutz, Slagter, Dunne, \& Davidson, 2008; Zylowska e cols., 2008).

Em suma, salientamos a importância de se conhecer uma realidade antes de planejar e propor uma intervenção. No presente estudo, foi observado através do grande número de pessoas que manifestaram interesse e conhecimento sobre o tema, independente de algumas características pessoais e de motivação, que a meditação pode se constituir como uma forma de intervenção destinada aos alunos universitários da UFRGS. Intervenções como essa podem ser oferecidas como uma atividade aplicada de pesquisa e extensão, atendendo a diversas demandas e objetivos. Entre eles, o desenvolvimento emocional e cognitivo do aluno; a oportunidade para a integração de estudantes de distintas formações, estimulando o relacionamento, o aprendizado de algo novo de uma forma distinta daquela que estão acostumados em seus respectivos cursos; e ainda reforçar a importância que os próprios alunos atribuíram ao estudo científico, servindo, além de tudo que já foi apontado, como um estímulo ao interesse e ao gosto pela produção científica.

\section{Referências}

Baer, R. A. (Ed.). (2006). Mindfulness-Based Treatment Approaches: Clinician's Guide to Evidence Base and Applicantions. San Diego: Academic Press.

Bardin, L. (1977). Análise de Conteúdo. Lisboa: Edições 70.

Bolsoni-Silva, A. T., Loureiro, S. R., Rosa, C. F., \& Oliveira, M. C. F. A. (2010). Caracterização das habilidades sociais de universitários. Contextos Clínicos, 3(1), 62-75. 
Broderick, P. C., \& Stacie, M. (2009). Learning to BREATHE: A pilot trial of a mindfulness curriculum for adolescents. Advances in School Mental Health Promotion, 2(1), 35-46.

Cardoso, R., Souza, E., Camano, L., \& Leite, J. R. (2004). Meditation in health: An operational definition. Brain Research Protocols, 14, 58-60.

Cerchiari, E. A. N. (2004). Saúde mental e qualidade de vida em estudantes universitários. Tese de Doutorado, Universidade Estadual de Campinas, Campinas, São Paulo.

Domingos, N. A. M., \& Domingos, J. C. V. (2005). Levantamento sobre o uso de álcool e drogas em universitários. Revista Brasileira de Terapias Cognitivas, 1(1), 75-82.

Ekman, P., Davidson, R. J., Ricard, M., \& Wallace, B. A. (2005). Buddhist and psychological perspectives on emotions and wellbeing. Current Directions in Psychological Science, 14(2), 59-63.

Flook, L., Smalley, S. L., Kitil, M. J., Galla, B. M., Kaiser-Greenland, S., Locke, J., \& cols. (2010). Effects of mindful awareness practices on executive functions in elementary school children. Journal of Applied School Psychology, 26(1), 70-95.

Gastaud, M. B., Souza, L. D. M., Braga, L., Horta, C. L., Oliveira, F. M., Sousa, P. L. R., \& cols. (2006). Bem-estar espiritual e transtornos psiquiátricos menores em estudantes de psicologia: estudo transversal. Revista de Psiquiatria do Rio Grande do Sul, 28(1), 12-18.

Harding, T. W., Arango, M. V., Baltazar, J., Climent, C. E., Ibrahim, H. H. A., Ignacio, L. L. \& cols. (1980). Mental Disorders in primary health care: a study of their frequency and diagnosis in four development contries. Psychological Medicine, 10, 231-241.

Institute, M. L. (2010). Education, Developmental Neuroscience and Contemplative Practices: Questions, Challenges, and Opportunities. Recuperado: 01 jun 2011. Disponível: http://www. mindandlife.org/pdfs/sri_conference_brochure_2010.pdf

Jain, S., Shapiro, S. L., Swanick, S., Roesch, S. C., Mills, P. J., Bell, I., \& cols. (2007). A randomized controlled trial of mindfulness meditation versus relaxation training: effects on distress, positive states of mind, rumination and distraction. Annals of Behavioral Medicine, 33(1), 11-21.

Khalsa, S. B., Hickey-Schultz, L., Cohen, D., Steiner, N., \& Cope, S. (2011). Evaluation of the mental health benefits of yoga in a secondary school: A preliminary randomized controlled trial. The Journal of Behavioral Health Services \& Research, Jun(7).

Lantieri, L. \& Goleman, D. J. (2008). Building emotional intelligence: Techniques to cultivate inner strength in children. Boulder: Sounds True.
Leite, J. R., Leite, F., Ornellas, M., Amemiya, T. M., Almedia, A. A. F., Dias, A. A., Afonso, R. et al. (2010). Effect of progressive selffocus meditation on attention, anxiety, and depression scores. Perceptual and Motor Skills, 110(3), 840-848.

Lima, M. C. P., Domingues, M. S. \& Cerqueira, A. T. A. R. (2006). Prevalência e fatores de risco para transtornos mentais comuns entre estudantes de medicina. Revista de Saúde Pública, 40(6), 1035-1041.

Lutz, A., Dunne, J. D., \& Davidson, R. J. (2007). Meditation and the neuroscience of consciousness: An introduction. Em P. Zelazo, M. Moscovitch \& E. Thompson (Eds.), Cambridge Handbook of Consciousness (pp. 499-554). New York: Cambridge University Press.

Lutz, A., Slagter, H. A., Dunne, J. D., \& Davidson, R. J. (2008). Attention regulation and monitoring in meditation. Trends in Cognitive Sciences, 12(4), 163-169.

Manjunath, N. K., \& Shirley, T. (2004). Spatial and verbal memory test scores following yoga and fine arts camps for school children. Indian Journal of Physiology and Pharmacology, 48(3), 353-356.

Mari, J., \& Willians, P. A. (1986). A validity study of a psychiatric screening questionnaire (SRQ-20) in primary care in the city of São Paulo. British Journal of Psychiatry, 148, 23-26.

McCrae, R., Martin, T., \& Costa, P. (2005). Age trends and age norms for the NEO Personality Inventory-3 in adolescents and adults. Assessment, 12(4), 363-373.

Mendelson, T., Greenberg, M. T., Dariotis, J. K., Gould, L. F., Rhoades, B. L., \& Leaf, P. J. (2010). Feasibility and preliminary outcomes of a school-based mindfulness intervention for urban youth. Journal of Abnormal Child Psychology, 38(7), 985-994.

Menezes, C. B., \& Dell'Aglio, D. D. (2009). Por que meditar? Os benefícios percebidos da prática de meditação. Psicologia em Estudo, 14(3), 565-573.

Menezes, C. B., \& Dell'Aglio, D. D. (2009). Os efeitos da meditação à luz da investigação científica em psicologia: Revisão de literatura. Psicologia: Ciência e Profissão. 29(2), 276-289.

Menezes, C. B., \& Dell'Aglio, D. D. (2010). The relationship between the practice of sitting and silent meditation, and psychological wellbeing, and the effects of personality traits. Interamerican Journal of Psychology, 44(1), 140-149.

Oliveira, M. L. C., Dantas, C. R., Azevedo, R. C. S., \& Banzato, C. E. M. (2008). Demographics and complaints of university students who sought help at a campus mental health service between 1987 and 2004. Sao Paulo Medical Journal, 126(1), 58-62.

Shapiro, S. L., Brown, K. W., Astin, J. A., \& Duerr, M. (2008). Toward the integration of meditation into higher education: A review of 
research. Recuperado: 20 jun 2011. Disponível: http://www. contemplativemind.org/programs/academic/MedandHigherEd.pdf

Shapiro, S. L., Oman, D., Thoresen, C. E., Plante, T. G., \& Flinders, T. (2008). Cultivating mindfulness: Effects on well-being. Journal of Clinical Psychology, 64(7), 840-862.

Tanner, M. A., Travis, F., Gaylord-King, C., Haaga, D. A. F., Grosswald, S., \& Schneider, R. H. (2009). The effects of the transcendental meditation program on mindfulness. Journal of Clinical Psychology, 65(6), 574-589

Teixeira, M. A. P., \& Gomes, W. B. (2004). Estou me formando... e agora? Reflexões e perspectivas de jovens formandos universitários. Revista Brasileira de Orientação Profissional, 5(1), 47-62.
Wallace, B. A., \& Shapiro, S. L. (2006). Mental balance and well-being: building bridges between buddhism and western psychology. American Psychologist, 61(7), 690-701.

Walsh, R., \& Shapiro, S. L. (2006). The meeting of meditative disciplines and western psychology: A mutually enriching dialogue. American Psychologist, 61(3), 227-239.

Zylowska, L., Ackerman, D. L., Yang, M. H., Futrell, J. L., Horton, N. L., Hale, T. S. e cols. (2008). Mindfulness meditation training in adults and adolescents with ADHD: A feasibility study. Journal of Attention Disorders, 11(6), 737-746.
Recebido em: 17/10/2011

Reformulado em: 22/04/2012

Aprovado em: 23/07/2012

\section{Sobre as autoras}

Carolina Baptista Menezes (menezescarolina@hotmail.com)

Doutoranda no Laboratório de Psicologia Experimental, Neurociência e Comportamento, Departamento de Psicologia do Desenvolvimento e Personalidade, Universidade Federal do Rio Grande do Sul, Mestre em Psicologia pela mesma instituição. Endereço: Rua Ramiro Barcelos, 2600, sala 105, Porto Alegre, RS. 90035-003, Brasil.

Bruna Fiorentin (bubafiorentin@hotmail.com)

Auxiliar de pesquisa no Laboratório de Psicologia Experimental, Neurociência e Comportamento, Departamento de Psicologia do Desenvolvimento e Personalidade, Universidade Federal do Rio Grande do Sul, aluna de graduação em Psicologia pela mesma instituição. Endereço: Rua Ramiro Barcelos, 2600, sala 105, Porto Alegre, RS. 90035-003, Brasil.

Lisiane Bizarro (lisiane.bizarro@gmail.com)

Professora e Coordenadora do Laboratório de Psicologia Experimental, Neurociência e Comportamento, Departamento de Psicologia do Desenvolvimento e Personalidade, Universidade Federal do Rio Grande do Sul, Pós-doutora. Endereço: Rua Ramiro Barcelos, 2600, sala 105, Porto Alegre, RS. 90035-003, Brasil.

Esta pesquisa tem o apoio do Conselho Nacional de Desenvolvimento Científico e Tecnológico - CNPq. 